\title{
A universal wind profile for the inversion-capped neutral atmospheric boundary layer
}

\author{
Kelly, Mark C.; Cersosimo, Roberto Alessio; Berg, Jacob
}

Published in:

Quarterly Journal of the Royal Meteorological Society

Link to article, DOI:

10.1002/qj.3472

Publication date:

2019

Document Version

Peer reviewed version

Link back to DTU Orbit

Citation (APA):

Kelly, M. C., Cersosimo, R. A., \& Berg, J. (2019). A universal wind profile for the inversion-capped neutral atmospheric boundary layer. Quarterly Journal of the Royal Meteorological Society, 145(720), 982-992. https://doi.org/10.1002/qj.3472

\section{General rights}

Copyright and moral rights for the publications made accessible in the public portal are retained by the authors and/or other copyright owners and it is a condition of accessing publications that users recognise and abide by the legal requirements associated with these rights.

- Users may download and print one copy of any publication from the public portal for the purpose of private study or research.

- You may not further distribute the material or use it for any profit-making activity or commercial gain

- You may freely distribute the URL identifying the publication in the public portal

If you believe that this document breaches copyright please contact us providing details, and we will remove access to the work immediately and investigate your claim. 


\section{A universal wind profile for the inversion-capped neutral atmospheric boundary layer}

\section{Mark Kelly $^{1 *}$ | Roberto Alessio Cersosimo ${ }^{2}$ Jacob Berg $^{1}$}
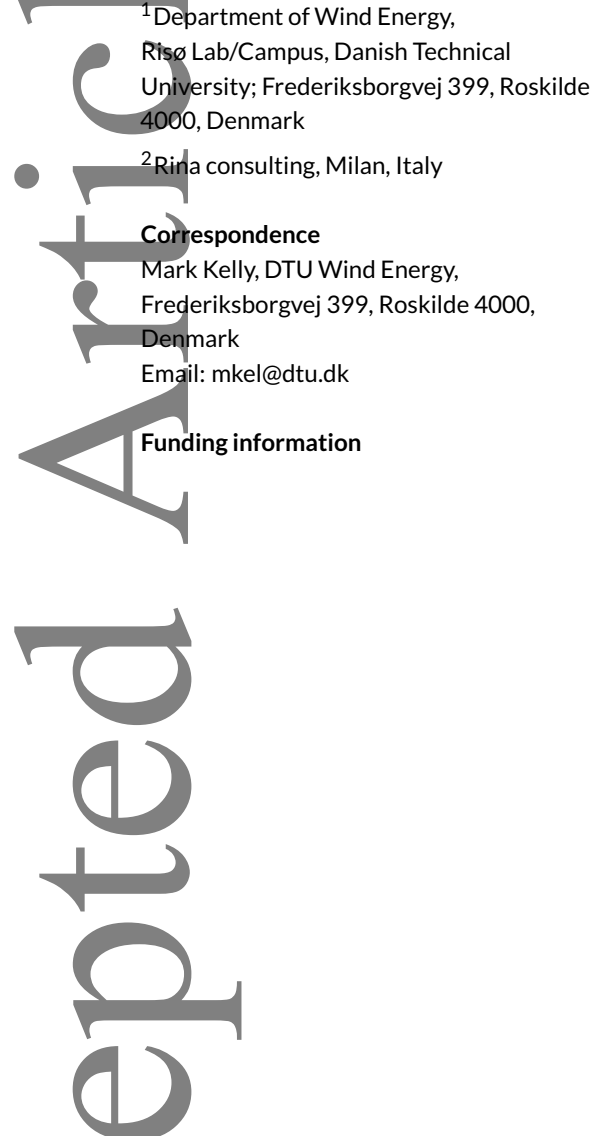

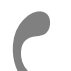

Abbreviations: ABL, atmospheric boundary layer; ASL, atmospheric surface layer; LES, large-eddy simulation; M-O, Monin-Obukhov;

MOST, Monin-Obukhov stability theory.

Wind profiles above the atmospheric surface layer are not accurately described by classic similarity theories. Far from the surface the underlying assumptions of such surfacelayer theories break down due to stronger influences of the buoyancy forces induced by the temperature inversion that caps the atmospheric boundary layer $(A B L)$, as well as the Coriolis force. This paper examines the influence of these forces on the mean flow and presents a new similarity theory to predict mean wind profiles in and above the surface layer, for an ABL with zero surface heat flux and capped by an inversion of potential temperature-i.e. the conditionally neutral $A B L$. The analysis here is based on the results of seventeen large-eddy simulations over a flat homogeneous rough surface, which leads to and supports the new similarity theory. The development is based on two applications of the Buckingham-Pi theorem. A first application allows determination of the entrainment-induced heat flux profile through the $A B L$ and into the surface layer, which is then used within a second dimensional argument for the vertical shear of mean wind speed. We subsequently find a new dimensionless group $\left(\Pi_{2}\right)$ depending on the cappinginversion strength, Coriolis parameter, surface stress, and

This article has been accepted for publication and undergone full peer review but has not been through the copyediting, typesetting, pagination and proofreading process, which may lead to differences between this version and the Version of Record. Please cite this article as doi: $10.1002 / \mathrm{qj} .3472$ 
ABL depth; it is correlated to the dimensionless shear $\left(\Pi_{1}\right)$ through a universal function $\beta$. Integrating the functional relation between $\Pi_{1}$ and $\Pi_{2}$, an equation for the mean wind speed profile is obtained: it effectively includes an additive 'correction' to the log-law in terms of $\Pi_{2}$, analogous to the Monin-Obukhov profile correction function. Unlike surfacelayer similarity, the new form accounts for the influences of both the surface and ABL top. Relative to LES the new profile form exhibits errors in mean wind speed below $5 \%$ for heights below $90 \%$ of the $\mathrm{ABL}$ depth; this is relevant for applications above the surface layer (e.g. wind energy).

\section{KEYWORDS}

similarity theory, capping inversion, stability, entrainment

\section{I INTRODUCTION}

In recent years, interest in wind profiles and description of the mean flow above the surface layer has increased. Driven bylapplications such as wind energy, where hub-heights of modern wind turbines typically exceed the depth of the atmospheric surface layer (ASL), there is a need for better characterization of the wind beyond the ASL. Above the ASL, accepted and classic similarity theories for the wind profile, namely Monin-Obukhov (M-O) theory, become less valid (e.g. Wyngaard, 1973; Gryning et al., 2007; Optis et al., 2014; Kelly and Troen, 2016); there is thus also a long-standing interest in consistent description of the wind profile at such heights (e.g. Blackadar, 1962; Hess and Garratt, 2002; Kelly et al., 2014a). M-O theory is defined for stationary conditions over flat homogeneous terrain, assuming that surface-layer parameters - which dictate the wind profile-do not vary appreciably (Foken, 2004) over the depth of the ASL (Horst, 1999; Wyngaard, 2010). The applicability of M-O theory becomes yet more tenuous in the upper part of the $A B L$, where other parameters affect the wind field.

Fon the upper part of the $A B L$ to be considered, one must address the influence of additional parameters: the potential temperature inversion magnitude and Coriolis parameter (e.g. Grachev et al., 2005; Zilitinkevich and Esau, 2005; Zilitinkevich et al., 2012). By potential temperature inversion we mean a positive gradient of mean potential temperature above the $A B L$, i.e. $(\partial \Theta / \partial z)_{c}>0$, where the subscript ' $c$ ' denotes capping inversion. This is often written in terms of the Brunt-Väisälä frequency $N_{c} \equiv \sqrt{\left(g / \theta_{0}\right)(\partial \Theta / \partial z)_{c}}$, sometimes referred to as the 'strength of the inversion.' The presence of a potential temperature inversion sets the height of the ABL (e.g. Deardorff, 1974; Wyngaard, 2010)

and induces a downward entrainment (flux) of higher potential temperature, which causes stable stratification within ABL (Tennekes and Driedonks, 1981; Zilitinkevich and Calanca, 2000).

Traditional classification of $A B L s$ is based on surface potential temperature flux $\left.Q_{0} \equiv \overline{w \theta}\right|_{z=0}$, and may lead to inaccurate description of mean profiles (of e.g. wind and temperature) when applied to boundary layers capped by inversions $(\partial \Theta / \partial z)_{c}$. To distinguish and allow for the effects of the inversion, a split of the neutral ABL classification was introduced by Zilitinkevich et al. (2012): 'truly neutral' and 'conditionally neutral' ABLs. A 'truly neutral' ABL is characterized by a constant mean potential temperature profile (neutral stratification), while a 'conditionally neutral' $A B L$ is capped by a positive gradient of potential temperature that extinguishes the turbulence (stable stratification). 
The influence of the Coriolis force is typically excluded in surface-based theories, disregarded under the assumption that close to the surface its contribution to scalar fields (and the mean wind speed $S$ ) is negligible. However, further from the surface the relative contribution to $S(z)$ of the Coriolis force increases, approaching a balance with the pressuregradient force, i.e. a (mean) geostrophic balance. The objective of the present work is to derive a similarity theory for the wind profile in conditionally neutral conditions. For this purpose seventeen large eddy simulations (LES) have been ed as input data for this study.

\section{I LARGE-EDDY SIMULATIONS}

The analysis here was done on data from seventeen different LES of the conditionally neutral ABL (i.e. $Q_{0}=0 \mathrm{Km} \mathrm{s}^{-1}$, $N_{c} \neq 0$ ), which were run using NCAR's standard LES code (Sullivan and Patton, 2011; Sullivan et al., 2016). The group of simulations covers a range of atmospheric parameters, in addition to the surface roughness length $z_{0}$ : the geostrophic wind speed $G$, Coriolis parameter $f$, and capping inversion strength $(\partial \Theta / \partial z)_{c}$ (or alternately $N_{c}$ ); here we aim to find the dependence of $S(z)$ upon these parameters. All the simulations are run in a domain of size [ $2560 \mathrm{~m} \times 2560 \mathrm{~m} \times 1792 \mathrm{~m}$ ] using $512^{3}$ grid points, and are initialised with a surface temperature $T_{0}=290 \mathrm{~K}$ and surface heat flux $\left(Q_{0}\right)$ of zero. ${ }^{1}$ The choice of the size of the domain and number of grid points were made to provide simulations within the 'high-accuracy zone' elucidated by Brasseur and Wei (2010).

In order to investigate mean profiles from the space-time dependent LES simulations, the results have been averaged in space (horizontal average in the $x-y$ plane) and time. To compare two different simulations, each must be analysed at the same dimensionless development time $(t / T)$, expressed here in terms of the turnover time $T_{t} \equiv z_{i} / u_{*}$ where $u_{*}$ is the (near-surface) friction velocity and $z_{i}$ is the ABL depth (defined by the maximum gradient as in Sullivan et al., 1998, where $\partial^{2} \Theta / \partial z^{2}=0$ ). The parameters of the simulations are summarised in Table 1, together with the derived statistics $\left\{z_{i}, u_{*}\right\}$ after time $50 T_{t}$.

To begin with an example, the mean wind speed profile for simulation $A$ is plotted in terms of dimensionless height, $S\left(z / z_{i}\right)$, in Figure 1 together with the logarithmic profile. Figure 1 may be seen as visualisation of the problem stated in the introduction: the presence of a capping inversion influences the mean wind speed profile within the ABL.

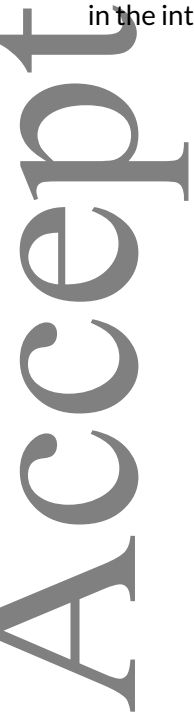

\footnotetext{
${ }^{1}$ The initial profile is not particularly relevant, as we are concerned with the stationary conditions after spin-up (see e.g. Pedersen et al., 2014).
} 
TAB LE 1 Set-up of LES runs; all have $n_{x}=n_{y}=n_{z}=512$ and zero surface heat flux $\left(Q_{0}=0 \mathrm{Km} \mathrm{s}^{-1}\right)$.

\begin{tabular}{|ccccc|cc}
\hline case & $z_{0}[\mathrm{~m}]$ & $G\left[\mathrm{~m} \mathrm{~s}^{-1}\right]$ & $\left.\frac{\partial \Theta}{\partial z}\right|_{C}\left[\mathrm{Km}^{-1}\right]$ & $f\left[\mathrm{~s}^{-1}\right]$ & $z_{i}[\mathrm{~m}]$ & $u_{*}\left[\mathrm{~m} \mathrm{~s}^{-1}\right]$ \\
\hline $\mathrm{A}$ & 0.05 & 10 & 0.003 & 0.0001 & 620 & 0.41 \\
$\mathrm{~B}$ & 0.05 & 5 & 0.003 & 0.0001 & 346 & 0.22 \\
$\mathrm{C}$ & 0.05 & 5 & 0.006 & 0.0001 & 273 & 0.22 \\
$\mathrm{D}$ & 0.1 & 5 & 0.003 & 0.0001 & 363 & 0.24 \\
$\mathrm{~F}$ & 0.1 & 10 & 0.003 & 0.0001 & 646 & 0.44 \\
$\mathrm{G}$ & 0.1 & 5 & 0.006 & 0.0001 & 288 & 0.23 \\
$\mathrm{H}$ & 0.05 & 10 & 0.006 & 0.0001 & 489 & 0.41 \\
$\mathrm{I}$ & 0.1 & 10 & 0.006 & 0.0001 & 512 & 0.43 \\
$\mathrm{~J}$ & 0.1 & 10 & 0.006 & 0.00005 & 548 & 0.35 \\
$\mathrm{~K}$ & 0.1 & 10 & 0.003 & 0.00005 & 705 & 0.38 \\
\hline $\mathrm{L}$ & 0.05 & 5 & 0.003 & 0.00005 & 382 & 0.20 \\
\hline $\mathrm{M}$ & 0.05 & 5 & 0.006 & 0.00005 & 299 & 0.18 \\
$\mathrm{~N}$ & 0.1 & 5 & 0.003 & 0.00005 & 401 & 0.21 \\
$\mathrm{O}$ & 0.1 & 5 & 0.006 & 0.00005 & 310 & 0.19 \\
$\mathrm{P}$ & 0.05 & 10 & 0.003 & 0.00005 & 676 & 0.36 \\
$\mathrm{Q}$ & 0.05 & 10 & 0.006 & 0.00005 & 530 & 0.34 \\
\hline $\mathrm{R}$ & 0.2 & 5 & 0.003 & 0.0001 & 383 & 0.25 \\
\hline & & & & & & \\
\hline
\end{tabular}

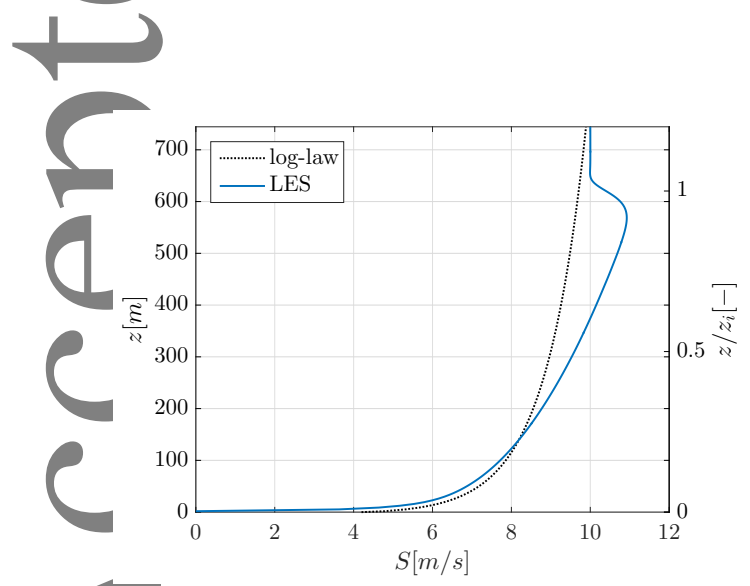

FIGURE 1 Comparison between wind profile of simulation A (solid) and logarithmic profile (dotted)

Close to the surface the log-law provides a good prediction of the LES mean wind speed profile, but moving upwards

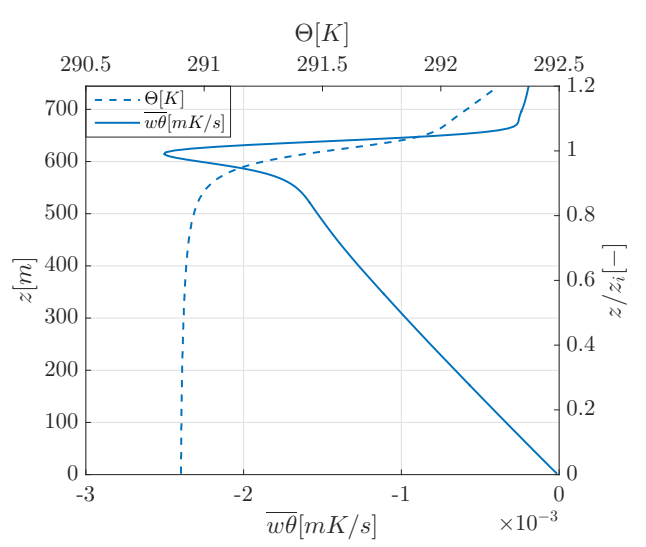

FIGURE 2 Profiles of mean potential temperature $\Theta$ and flux $\overline{w \theta}$ for simulation $A$ 
in the $A B L$ the two profiles deviate significantly: predicting the speed profile with the log-law may lead to errors exceeding $25 \%$ for the cases analysed here (shown later in Figure 15 for all cases). The potential temperature inversion $(\partial \Theta / \partial z)_{c}>0$ induces a mean temperature flux $\overline{w \theta}(z)<0$ (i.e. downward flux of warmer air), as shown in Figure 2; this reduces the turbulent kinetic energy and significantly affects the wind profile $S(z)$.

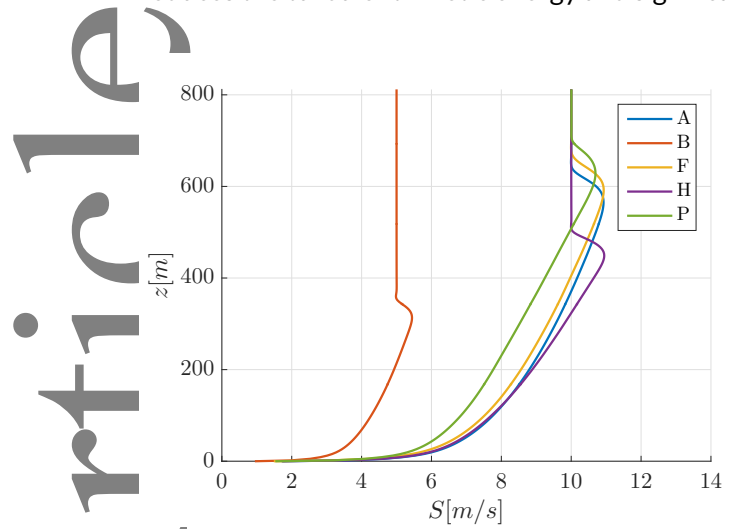

FIGURE 3 Mean wind speed profiles for simulations $\mathrm{A}, \mathrm{B}, \mathrm{F}, \mathrm{H}$ and $\mathrm{P}$

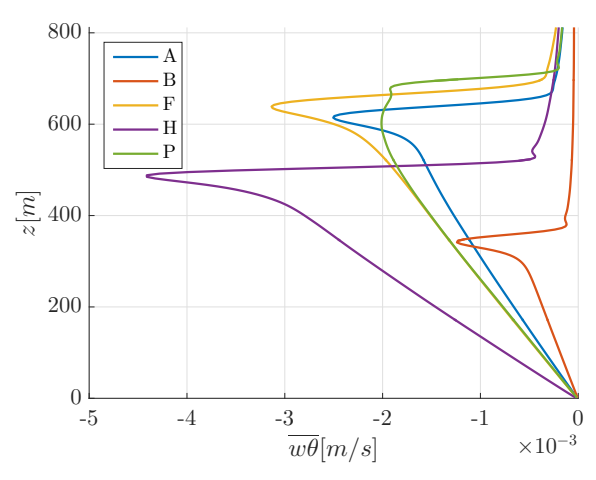

FIGURE 4 Potential temperature flux profiles for simulations $\mathrm{A}, \mathrm{B}, \mathrm{F}, \mathrm{H}$ and $\mathrm{P}$

\begin{abstract}
The collective influence of the parameters $\left\{G, f,(\partial \Theta / \partial z)_{c}, z_{0}\right\}$ upon $S(z)$ and $\overline{w \theta}(z)$ diagnosed from the LES is
shown in Figures 3 and 4 . From these figures, one sees the influence of each:
\end{abstract}

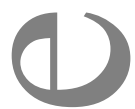

1.) Higher geostrophic wind $G$ causes larger shear stresses at the surface, which result in taller $A B L$ and larger $\overline{w \theta}$.

2. Increased $(\partial \Theta / \partial z)_{c}$, i.e. stronger inversions, causes more negative (increased amplitude) $\overline{w \theta}(z)$ and a shallower $A B L$.

3. Larger $f$ results in slightly smaller magnitude of $\overline{w \theta}(z)$ and a slightly shallower ABL. The functional behaviour is sensitive to $f$, and is particularly different close to the inversion.

4. Larger $z_{0}$, i.e. rougher surface, causes higher shear stresses at the surface ( $u_{*}$ for a given $G$ ); this results in a taller $A B L$ and larger magnitude of $\overline{w \theta}(z)$.

5. The qualitative trend of $S(z)$ appears to be similar in all the simulations and highly dependent on $\overline{w \theta}(z)$-and thus $(\partial \Theta / \partial z)_{c} ; S(z)$ is well-predicted by the log-law close to the surface, where $\overline{w \theta} \rightarrow 0$. Above the surface layer, where $\overline{w \theta}$ is more negative, the simulated $S(z)$ exceeds the log-law prediction. The wind speed increases up to a maximum (super-geostrophic jet) close to the ABL top and then adjusts to its geostrophic value as $z>\sim z_{i}$.

6. The profile $\overline{w \theta}(\mathrm{z})$ is linear for most of the $\mathrm{ABL}$, and $\partial \overline{w \theta} / \partial z$ appears to depend on $u_{*}(\partial \Theta / \partial z)_{c}$.

Points 1-5 above have been documented before in the literature, but point 6 has not yet been treated well; it is the focus of this paper, along with the relation between entrainment heat flux and $S(z)$. As analysed in detail in Chapter 3 and 4 of Cersosimo (2017), and to some extent in Pedersen et al. (2014), the effects listed above are caused by changes of the shear and buoyancy term in the turbulent kinetic energy budget of the flow. 


\section{3 | THEORY AND DEVELOPMENT: THE CONDITIONALLY NEUTRAL ABL}

The aim of this present section is to obtain a simple expression for the wind profile through similarity theory. This is composed by two applications of the Buckingham $\Pi$ theorem. The first application allows to predict the $\overline{w \theta}$ profile for most of the $A B L$ height. This profile is then used in a successive application of the theorem that enables prediction of the wind speed profile with accuracy up to the $90 \%$ of the ABL height.

\subsection{Potential temperature flux profile: $\overline{w \theta}(z)$}

As seen in Figure 4 the profile of $\overline{w \theta}(z)$ is linear for most of the boundary layer height and its slope depends on $u_{*}$ and $\left.\frac{\partial \Theta}{\partial z}\right|_{c}$ which suggests that these are significant parameters. This provided a first guess of the significant dimensional groups to be considered when applying Buckingham $\Pi$ theorem. Considering the following dimensional $n=5$ grouping: strength of the inversion $\left(\left.\frac{\partial \Theta}{\partial z}\right|_{c}\right)$, vertical coordinate $z$, surface stress $\tau_{0}$, air density $\rho$ and the potential temperature flux profile $\overline{w \theta}(z)$ and using the $m=4$ dimensions $[m, s, K g, K]$ follows that there will be only $n-m=1$ dimensionless group that describes the 5 parameters involved:

$$
\Pi_{\overline{w \theta}}=\left(\left.\frac{\partial \Theta}{\partial z}\right|_{c}\right)^{\alpha} z^{\beta} \tau_{0}^{\gamma} \rho^{\delta} \cdot \overline{w \theta}
$$

Consideration of units results in $\alpha=\beta=-1, \gamma=-1 / 2$, and $\delta=1 / 2$; recalling that $\tau_{0}=\rho u_{*}^{2}$ then gives a heat flux profile defined by

$$
\overline{w \theta}(z)=\left.\Pi_{w \theta} u_{*} z \frac{\partial \Theta}{\partial z}\right|_{c}
$$

This follows the classic flux-gradient form $\overline{w \theta}=-K \partial \Theta / \partial z$ (where $K$ is the product of turbulent velocity and length scales, e.g. Wyngaard, 2010), and yet more closely resembles the surface-layer form $\overline{w \theta}(z)_{\mathrm{ASL}}=-k u_{*} z \partial \Theta / \partial z$; here in (2) the negative sign is absorbed into $\Pi_{\overline{w \theta}}$. The formulation (2) is consistent with the observation that in the conditionally neutral $A B L$ the potential temperature flux is zero at the surface and observed to be linearly increasing with height through most of the boundary layer (see Figure 4). Thus over the range of $z$ where (2) is valid, then $\Pi_{\overline{w \theta}}$ has to be a constant. Expressing $\overline{w \theta} \simeq m_{\overline{w \theta}} z$, where $m_{\overline{w \theta}} \equiv \partial \overline{w \theta} / \partial z$ is simply the 'slope' of the flux profile (the heat flux divergence in horizontally homogeneous conditions), then

$$
\Pi_{\overline{w \theta}}=\frac{z(\partial \overline{w \theta} / \partial z)}{\left.u_{*} z \frac{\partial \Theta}{\partial z}\right|_{c}}=\frac{m_{\overline{w \theta}}}{\left.u_{*} \frac{\partial \Theta}{\partial z}\right|_{c}}
$$

To check the validity of assuming a linear profile and to obtain the value of the corresponding constant $\Pi_{\overline{w \theta}}$, the slope of the linear part of $\overline{w \theta}(z)$ is plotted versus the corresponding values of $\left.u_{*}(\partial \Theta / \partial z)\right|_{c}$ in Figure 5 . There it can be observed that indeed (3) holds, giving $\Pi_{\overline{w \theta}}=-0.0031$ and confirming the initial assumption of constant $\partial \overline{w \theta} / \partial z$. Though the agreement is generally good, it can be seen in Figure 5 that the effect of the Coriolis parameter and roughness length is not perfectly captured; this causes errors in prediction of $\partial \overline{w \theta} / \partial z$ up to $20 \%$, as shown in Table 2 .

In order to account for the influence of the Coriolis parameter, two approaches can be followed. One approach would be to include an additional parameter $(f)$ within application of the Buckingham $n$ theorem. However, this would result in an additional dimensionless group, and an additional universal functional relation between the two dimensionless groups would have to be found. For the sake of simplicity the dependence on $f$ has been included through 


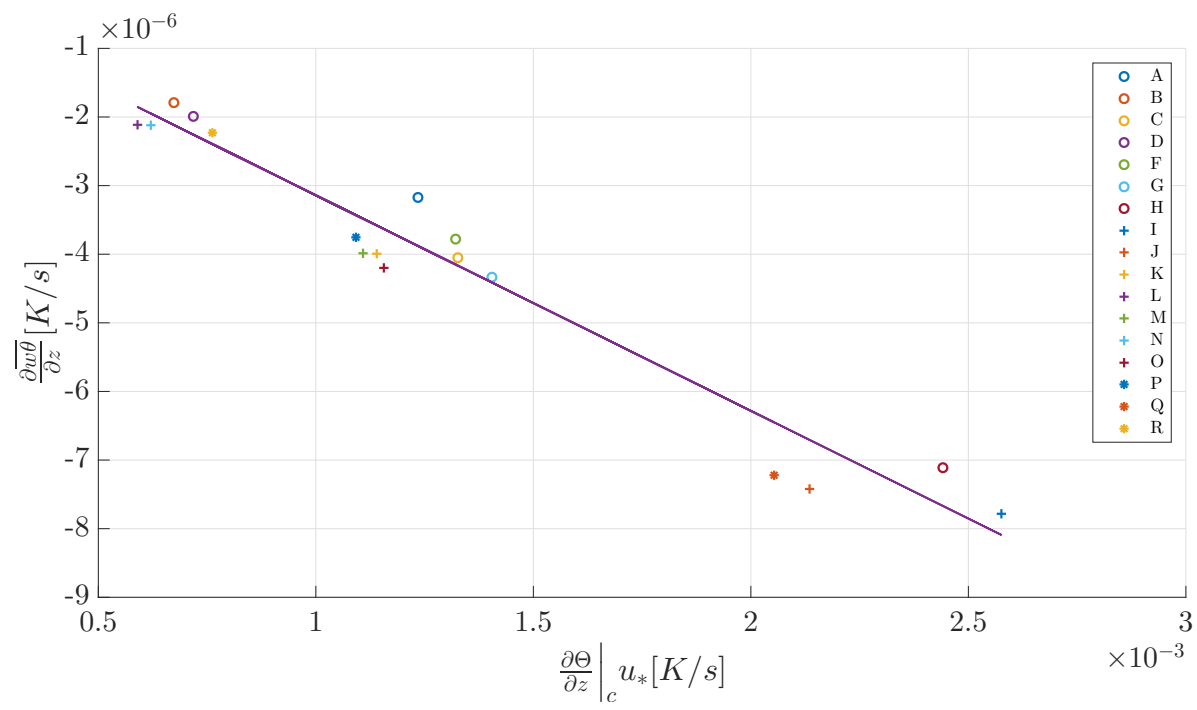

FIGURE 5 Dimensional analysis on $\overline{w \theta}$ : convergence of $\Pi_{\overline{w \theta}}$ to a constant

TAB LE 2 Error $(\epsilon)$ in prediction of $\partial \overline{w \theta} / \partial z$, using $\Pi_{\overline{w \theta}}$ (eqn. 3 without Ro) or via $\Pi_{\overline{w \theta} 2}$ (eqn. 4 including Ro effect).

\begin{tabular}{l|ccccccccccccccccc}
\hline & $\mathrm{A}$ & $\mathrm{B}$ & $\mathrm{C}$ & $\mathrm{D}$ & $\mathrm{F}$ & $\mathrm{G}$ & $\mathrm{H}$ & $\mathrm{I}$ & $\mathrm{J}$ & $\mathrm{K}$ & $\mathrm{L}$ & $\mathrm{M}$ & $\mathrm{N}$ & $\mathrm{O}$ & $\mathrm{P}$ & $\mathrm{Q}$ & $\mathrm{R}$ \\
\hline$\epsilon_{\overline{\mathrm{w \theta}}}(\%)$ & 21 & 19 & 4 & 12 & 10 & 2 & 7 & 3 & -8 & -11 & -11 & -13 & -7 & -11 & -9 & -11 & 7 \\
$\epsilon_{\Pi_{\bar{w} \boldsymbol{}}}(\%)$ & 8 & 4 & -1 & -0.4 & -1 & -3 & 2 & -2 & -1 & -8 & -9 & -5 & -6 & -4 & -5 & -3 & -4 \\
\hline
\end{tabular}

\section{I}

a dimensionless correction based on a Rossby number Ro:

$$
\Pi_{\overline{w \theta 2}}=\Pi_{\overline{w \theta}} \operatorname{Ro}^{-0.3}=\frac{\partial \overline{w \theta} / \partial z}{\left.u_{*}(\partial \Theta / \partial z)\right|_{c}} \operatorname{Ro}^{-0.3}
$$

where the Rossby number here is defined as $\operatorname{Ro} \equiv u_{*} /\left(f z_{i}\right)$. The exponent $(-0.3)$ has been found empirically, to minimize the mean absolute error in prediction of $m_{\overline{w \theta}}$. Using (4) and plotting $\partial \overline{w \theta} / \partial z$ versus $\left.u_{*}(\partial \Theta / \partial z)\right|_{c} R_{0}^{0.3}$ as shown in Figure 6, the data collapse to give

$$
\frac{\partial \overline{w \theta}}{\partial z}=-\left.0.0016 u_{*} \frac{\partial \Theta}{\partial z}\right|_{c} R^{0.3}
$$

i.e. $\Pi_{\overline{w \theta} 2}=-0.0016$. We also note that one could alternately keep the coefficient of $\Pi_{\overline{w \theta}}=-0.0031$ found from (3), and instead introduce a factor of $\left(\Pi_{\overline{w \theta} 2} / \Pi_{w \theta}\right)^{1 / 0.3} \approx 0.11$ within Ro in eq. 5); this is consistent, on average, with both (3) and known parameterizations for the ABL depth: $z_{i}$ can be estimated by $z_{i}=c_{h} u_{*} / f$, where $c_{h} \sim 0.1-0.2$ for the range of $z_{i}$ and $N_{c}$ considered (Zilitinkevich and Esau, 2002), so on average the Ro ${ }^{0.3}$ defined here would roughly cancel this factor of 0.11 . Employing (4)-(5) leads to significantly reduced prediction errors compared to ignoring Ro, as reported in Table 2. 


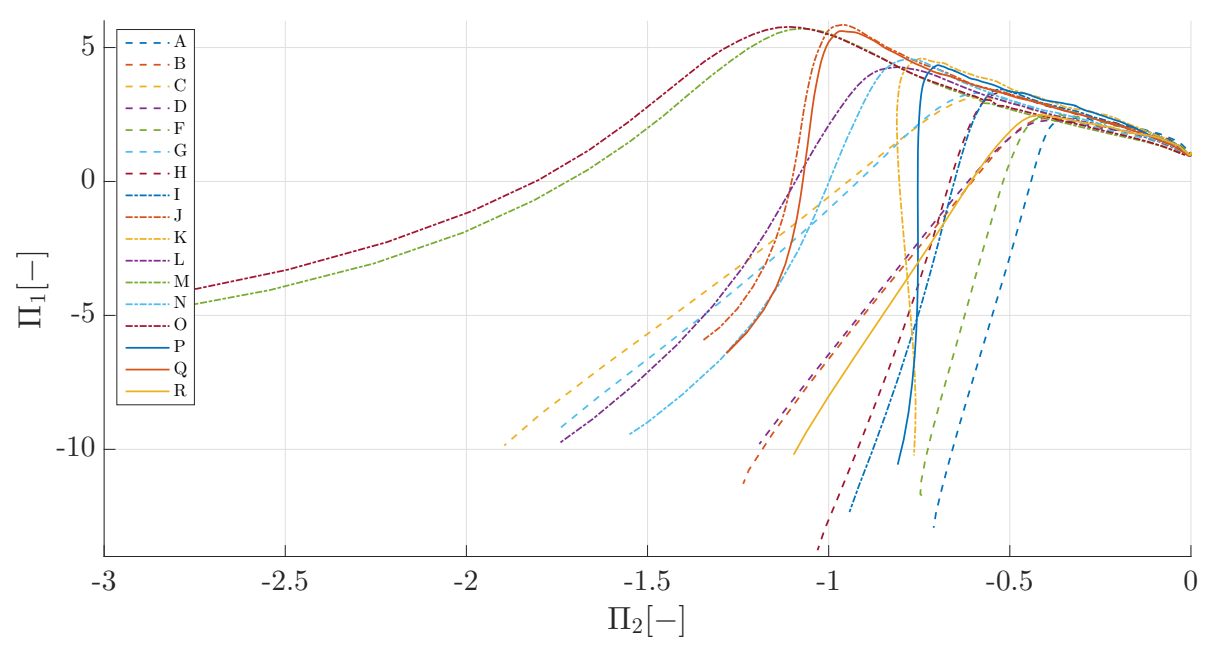

FIGURE $9 \quad \Pi_{1}$ versus $\Pi_{2}$ for all LES runs, over entire ABL

some negative value, where it starts diverging. The point where each case's function $\Pi_{1}=\beta\left(\Pi_{2}\right)$ starts to diverge from a constant $\beta$ corresponds to a height $z$ where the influence of the potential temperature inversion starts to dominate, so this observation sets the limits of the validity of the similarity theory developed. This can be observed in Figure 10, where the correlation between $\Pi_{1}$ and $\Pi_{2}$ is plotted as far up as the influence of the ABL top can be considered negligible; empirically we choose this to be $z / z_{i}=0.7$, which also ensures a linear profile of the potential temperature flux up to $z=0.7 z_{i}$.

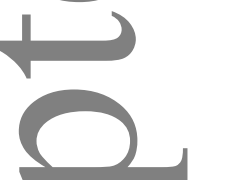

In Figure 10 it can be observed that for large magnitudes of $\Pi_{2}$ (above the ASL and/or for sufficiently large values of $\overline{w \theta}$ ), all the cases exhibit a linear behaviour with approximately the same slope, while for small values of $\Pi_{2}$ (in the ASL) the lines curve towards lower values of $\Pi_{1}$ and intersect the axis $\Pi_{2}=0$ at $\Pi_{1}$. Even if all the functions show a similar profile, it can be seen that the correlation between the two dimensionless groups is not exactly the same for all the simulations, and this slight difference may question the existence of a universal function $\beta$ that correlates $\Pi_{1}$ and $\Pi_{2}$. However, dimensional analysis is not guaranteed to provide perfectly clean results, due to its nature of being an approximation of the dominant physical phenomena based on the most significant dimensional groups representing the system. Furthermore, even if the LES simulations represent a powerful tool for the analysis of atmospheric flow, their accuracy may be questioned, especially close to the surface and inversion (Otte and Wyngaard, 2001). Keeping in mind these two last considerations, it has been concluded that the assumptions made regarding the significant dimensional groups are valid for the purposes of this paper-since the key developments here have most impact (are valid) above the ASL and below $0.7 z_{i}$. Up to this point it has been shown that, if the potential temperature flux profile $\overline{w \theta}(z)$ is used rather then $Q_{0}$, the dimensional groups of $\mathrm{M}-\mathrm{O}$ similarity represent a reasonable choice for conditionally neutral atmospheric boundary layers. 


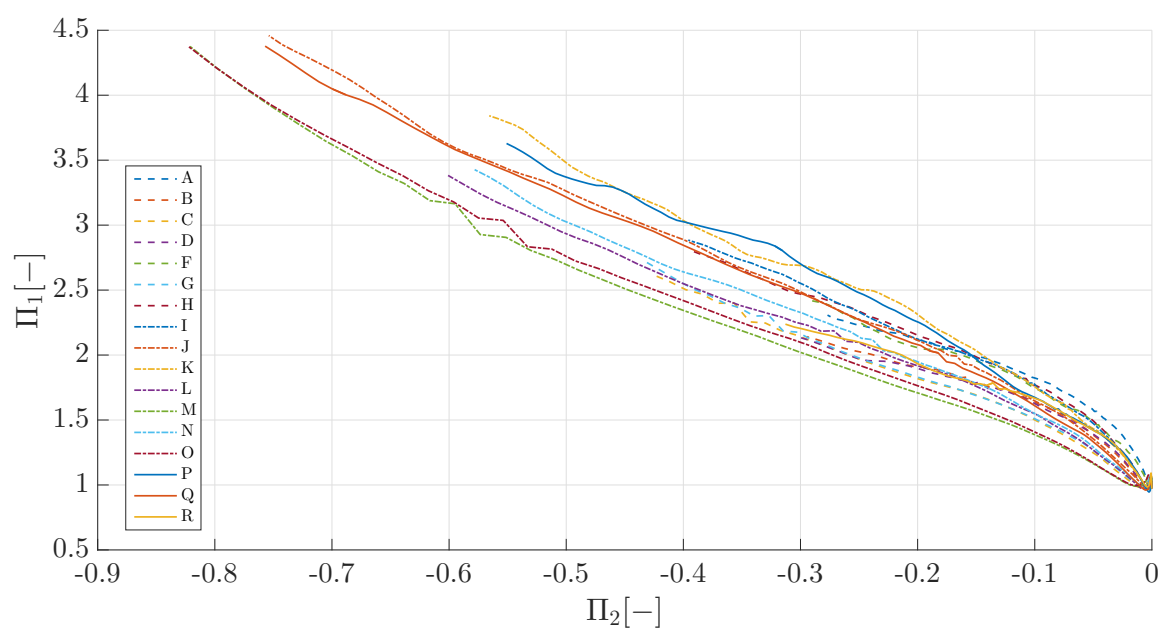

FIGURE 10 Support of $\beta$ function (9): $\Pi_{1}$ vs. $\Pi_{2}$ for heights up to $0.7 z_{i}$

\section{3 | Top-down similarity and wind profile}

Here we derive the mean wind profile following from the similarity form (9) and 'top-down' heat flux profile (6). Inserting (6) into (9) one can re-rewrite $\Pi_{2}$ as

$$
\Pi_{2}=\left.\Pi_{\overline{w \theta} 2} \frac{z\left(g / \theta_{0}\right)}{u_{*}^{3}} \frac{\partial \Theta}{\partial z}\right|_{c} u_{*} z \mathrm{Ro}^{0.3}=-\left(\frac{z}{\ell_{\mathrm{TD}}}\right)^{2}
$$

where we define the 'top-down' length scale by

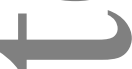

$$
\ell_{\mathrm{TD}} \equiv \sqrt{\frac{u_{*}^{2}}{-\left.\Pi_{\overline{w \theta}} \frac{g}{\theta_{0}} \frac{\partial \Theta}{\partial z}\right|_{c} \mathrm{Ro}^{0.3}}}=\frac{u_{*} / N_{c}}{0.04 \mathrm{Ro}^{0.15}}
$$

with $N_{c}$ being the Brunt-Väisälä frequency of the capping inversion. Note that $\ell_{\mathrm{TD}}$ could be compared to the "non-local external static-stability scale" of $u_{*} / N_{c}$ given by Zilitinkevich and Esau (2005), but modified to account for the effect of $\mathrm{ABL}$ depth and Coriolis parameter through the ABL Rossby number Ro $\equiv u_{*} /\left(f z_{i}\right)$, and with the mean efficiency of the entrainment process accounted for through the constant $\sqrt{-\Pi_{\bar{w} \theta 2}}=0.04$. Now using (10) and (11) the dimensionless shear (9) can be re-rwitten to show its explicit dependence on $z^{2} / \ell_{\mathrm{TD}}^{2}$ :

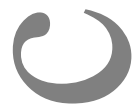

$$
\frac{k z}{u_{*}} \frac{d S}{d z}=\beta\left(\frac{-z^{2}}{\ell_{\mathrm{TD}}^{2}}\right)
$$

where we remind that the $\beta$-function is analogous to the MOST $\phi_{m}$-function, but accounting for the effectively stable conditions due to negative heat flux caused by the capping inversion.

As with Monin-Obukhov theory, the correlation between the two dimensionless groups can be re-written as a function of the vertical coordinate $z$ and a length scale (now $\ell_{\mathrm{TD}}$ ); this supports simplicity of application. The specific details of the linear function $\beta\left(\Pi_{2}\right)$ are found, and its applicability tested, by plotting dimensionless shear $\Pi_{1}$ versus $\Pi_{2}$ 


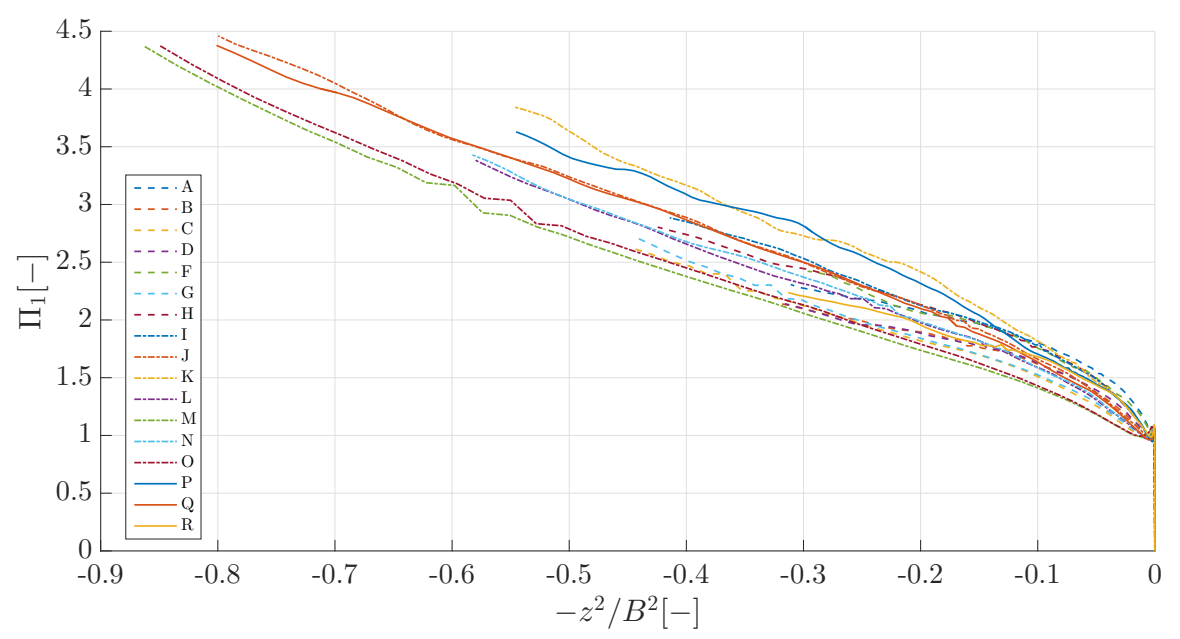

FIGURE 11 Dimensionless shear $\Pi_{1}$ versus $\Pi_{2}=-z^{2} / \ell_{T D}^{2}$, using (10)-(12).

employing (10)-(12). This is done in Figure 11, which shows that the functional behaviours presented in Figure 10 are matched by (12). All the cases present similar linear trends in terms of $z^{2} / \ell_{\mathrm{TD}}^{2}$ (Fig. 11), and therefore for each of them a line of the form

$$
\Pi_{1}=-a \frac{z^{2}}{\ell_{\mathrm{TD}}^{2}}+b
$$

was fitted to the data. Based on the assumptions made, we arrive at a single universal function $\beta\left(\Pi_{2}\right)$ :

$$
\beta\left(\frac{z^{2}}{\ell_{\mathrm{TD}}^{2}}\right)=-\langle a\rangle \frac{z^{2}}{\ell_{\mathrm{TD}}^{2}}+\langle b\rangle
$$

where $\langle a\rangle=-4.3$ and $\langle b\rangle=1$ are the mean of the parameters $a$ and $b$ respectively obtained by the fit of the single lines in Figure 11 over all cases. Inserting (14) into (12) we then obtain

$$
\frac{d S}{d z} \frac{k z}{u_{*}}=1-\langle a\rangle \frac{z^{2}}{\ell_{\mathrm{TD}}^{2}}
$$

simply $\Pi_{1}=1+\langle a\rangle \Pi_{2}=\beta\left(\Pi_{2}\right)$ in terms of dimensionless groups. Integrating (15) over $z$ and considering that the speed profile close to the surface is described well by the log-law (i.e. the integration constant is equal to $-\log z_{0}$ as in M-O theory), we find the wind speed profile $S(z)$ for the conventionally neutral boundary layer:

$$
\begin{aligned}
S(z) & =\frac{u_{*}}{k}\left[\log \left(\frac{z}{z_{0}}\right)-\frac{\langle a\rangle}{2}\left(\frac{z}{\ell_{\mathrm{TD}}}\right)^{2}\right] \\
& =\frac{u_{*}}{k} \log \left(\frac{z}{z_{0}}\right)+a_{B} \frac{N_{c}^{2} z^{2}}{k u_{*}} \mathrm{RO}^{0.3},
\end{aligned}
$$


- inclusion of the additional parameter Ro-which accounts for the effect of the Coriolis force-is justified in the dimensional analysis for the inversion-induced potential temperature flux (also mentioned in Cersosimo, 2017); and

- it is reasonable to assume that $\beta\left(z / \ell_{\mathrm{TD}}\right)$ is effectively a universal function, for homogeneous conditions with negligible surface heat flux and capping inversion with strength described by $N_{c}$.

Regarding applicability for the profile derived here, we note that approaching the equator $f \rightarrow 0$ and subsequently the $\mathrm{Ro}^{0.3}$ correction to the heat flux and wind profiles becomes large and non-physical. Thus we suggest a lower limit of $f$ based on applicability of geostrophic arguments (e.g. Arya and Wyngaard, 1974; Kelly et al., 2014b); following the European Wind Atlas (Troen and Petersen, 1989) and decades of its standard use, one expects the limit to be at the latitude where $f$ corresponds to $\sim 1 / 2-1$ day. This limit corresponds to where daily convective activity (on average) dominates boundary-layer depth development (moreso than any Coriolis effects), roughly at a latitude of 10 degrees.

One additional aspect regarding applicability, or uncertainty, stems from resolution-dependence of the large-eddy simulations used. In particular, although the turbulence and mean fields are essentially independent of resolution (within the high-accuracy zone of Brasseur and Wei, 2010, as noted earlier), the entrainment heat flux can have a slight dependence upon resolution, even with our relatively fine vertical resolution of $3.5 \mathrm{~m}$ (see e.g. Sullivan and Patton, 2011). But because the heat flux profile is linear in $(\partial \Theta / \partial z)_{c}$ and consequently linear in the associated entrainment flux $\langle w \theta\rangle_{\text {entrain }}$ (e.g. Otte and Wyngaard, 2001), for $z$ below $\sim 0.7 z_{i}$ at resolutions finer than $10-20 \mathrm{~m}$, then the small impact of (vertical) resolution is simply to affect the coefficient $\Pi_{\overline{w \theta} 2}$ in a similarly linear manner. In other words, the form (16) is the same independent of resolution, but there is a small uncertainty in the coefficient $a_{B}$ due to dependence of $\langle w \theta\rangle_{\text {entrain }}$ upon resolution; the uncertainty in $a_{B}$ is of similar magnitude to the entrainment-flux uncertainty, because $a_{B} \propto \Pi_{\overline{w \theta} 2}$ while $\Pi_{\overline{w \theta} 2} \propto\langle w \theta\rangle_{\text {entrain }}$; the slight (vertical) resolution dependence corresponds to a different simulated entrainment flux efficiency and consequently $\Pi_{\bar{w} \theta 2}$.

\section{(4) I concLusion}

Anew equation for the mean wind speed profile $S(z)$ in neutral, inversion-capped atmospheric boundary layers (the 'conventionally neutral' $A B L$ ) has been found through the Buckingham- $\Pi$ theorem. It can be written in a form comparable to $\mathrm{M}-\mathrm{O}$ similarity in stable conditions, as

$$
S(z)=\frac{u_{*}}{k}\left[\log \left(\frac{z}{z_{0}}\right)-\frac{\langle a\rangle}{2}\left(\frac{z}{\ell_{\mathrm{TD}}}\right)^{2}\right]
$$

where $\langle a\rangle=-4.3$ and

$$
\ell_{\mathrm{TD}}^{2}=\frac{\left(u_{*} / N_{c}\right)^{2}}{-\Pi_{\bar{w} \theta 2} \mathrm{Ro}^{0.3}}
$$

with $\Pi_{\overline{w \theta} 2} \simeq-0.0016$ and the ABL Rossby number defined by Ro $\equiv\left(u_{*} / f\right) / z_{i}$. Thus it is possible to predict the wind speed in the ABL over homogeneous terrain with zero surface heat flux, based on five parameters: the surface-layer friction velocity $u_{*}$, roughness length $z_{0}$, Coriolis parameter $f, A B L$ depth $z_{i}$, and free-stream Brunt-Väisälä frequency (inversion strength) $N_{c}=\left[\left(g / \theta_{0}\right)(\partial \Theta / \partial z)_{c}\right]^{1 / 2}$ where the latter may be expressed in terms of the capping inversion's mean temperature gradient $(\partial \Theta / \partial z)_{c}$ and the mean ABL temperature $\Theta_{0}$. For the LES simulations analysed, this new 
formulation returns a maximum error of $5 \%$, in contrast to errors growing beyond $25 \%$ when using the logarithmic profile, for heights up to $90 \%$ of the $A B L$ depth.

In practice three parameters $\left\{z_{0}, u_{*}, f\right\}$ are typically readily available; the $A B L$ depth $z_{i}$ can be a bit more challenging, though obtainable to some extent through mesoscale modelling (e.g. WRF) or via statistical estimates (Liu and Liang, 2010) and parameterizations (Zilitinkevich et al., 2012). Getting the inversion strength $N_{c}$ can be more difficult, as RASS observations (Emeis et al., 2012) are not commonly available, and models such as WRF that predict $N_{c}$ also offer limited reliability (e.g. García-Díez et al., 2013). We can loosely recommend a range of $(\partial \Theta / \partial z)_{c}$ similar to what was used here (3-6 K/ km), consistent with the 'typical midlatitude values' of 1-5 K/ km tabulated in Hartmann (1994) and values of $1-10 \mathrm{~K} / \mathrm{km}$ used by Sorbjan (1996), while also accounting for the modestly increased $(\partial \Theta / \partial z)_{c}$ just within the capping part (Pedersen et al., 2013) of the inversion, in contrast with the free-stream $\partial \Theta / \partial z$.

The form derived here could potentially be extended to include non-zero surface heat fluxes; however, need for this and its derivation are not clear for convective boundary layers (positive surface heat flux), where the entrainment effect is confined to the top portion of the $A B L$ due to the existence of the mixed layer stemming from convective mixing (e.g. Wyngaard, 2010). In stable boundary layers the heat flux profile described here could in principle be 'added' to that stemming from negative surface heat flux, though this is beyond the scope of the current paper. Future work also includes testing the formulation over a yet broader range of $f, z_{0}, N_{c}$, and $G$ (and subsequently potentially different values of $z_{i}$ and $u_{*}$ ), as well as comparing to LIDAR observations well above the surface layer. Another aspect regarding applicability for the profile derived here involves the range of $f$ : we have suggested a lower limit of $f$, and consequently latitude $\left(\sim 10^{\circ}\right)$ based on applicability of geostrophic arguments. Further, it is possible that a slightly different combination of $f$ and $z_{0}$ could be more optimal than Ro; both analysis of broader LES datasets, as well as comparison with observations, can justify the current formulation or facilitate adjustment.

Finally, a parallel development of the wind direction profile is underway, including connection with the theory presented herein.

\section{SUPPORTING INFORMATION}

Additional figures, showing the results for all LES cases analysed, may be found online as supplementary material. Whereas the main text here shows the heat flux profile, wind speed profile, and speed prediction error profile for case ' $A$ ' in Figures 7, 12, and 13 respectively (along with the wind speed error for all cases plotted together in Fig. 14), the supporting material includes these profiles for each LES case.

\section{ACKNOWLEDGEMENTS}

The authors are are grateful to Ned Patton and Peter Sullivan from the National Center for Atmospheric Research (Boulder, CO, USA), for sharing the LES code.

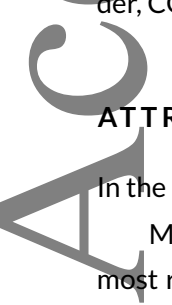

\section{ATTRIBUTION}

In the interest of open communication, the work can be attributed roughly as follows.

M.K. originated the concept; proposed the Ro-dependence; related the developments to other works, including most references; reformulated the derivations/equations, connecting to extant theory; interpreted the equations and explained the results; gave conditions for use; developed the uncertainty aspect and implications, via analytical forms; described LES usage, limitations and resolution; addressed input parameter issues, recommending values and 
connecting to literature; expressed range of applicability of results/theory; (re-)wrote abstract; made supplementary material section and graphical table of contents; created/wrote responses to reviewers.

R.A.C. analyzed the LES data; derived heat flux profile; derived form for the wind profile; created all plots; fitted dimensionless heat flux and wind speed gradients, finding empirical constants to relate the dimensionless groups; made article 'skeleton' including introduction to similarity theory, equations, plots, and comparisons of derived profile to lassic theory.

JB set up and ran the numerous large-eddy simulations (LES); discussed the concepts and progress along the way; suggested changes to the manuscript, including some references.

\section{REFERENCES}

Arya, S. P. S. and Wyngaard, J. C. (1974) Effect of baroclinicity on wind profiles and the geostrophic drag law for the convective Joundary layer. J. Atmos. Sci., 32, 767-778.

Blackadar, A. K. (1962) The vertical distribution of wind and turbulent exchange in a neutral atmosphere. J. Geophys. Res., 67, 3095--3102.

Brasseur, J. G. and Wei, T. (2010) Designing large eddy simulation of the turbulent boundary layer to capture law-of-the-wall scaling. Phys. Fluids, 22, 1-21.

Cersosimo, R. A. (2017) Effect of atmospheric boundary layer top and capping inversion properties on mean wind profiles. Master's thesis, Danske Tekniske Universitet, Department of Wind Energy Frederiksborgvej 3992800 Kgs. Lyngby Denmark.

Deardorff, J. W. (1974) Three-dimensional numerical study of the height and mean structure of a heated planetary boundary layer. Boundary-layer Meteorology, 7, 81-106, 81-106.

Emeis, S., Schaefer, K., Muenkel, C., Friedl, R. and Suppan, P. (2012) Evaluation of the interpretation of ceilometer data with RASS and radiosonde data. Boundary-layer Meteorology, 143, 25-35.

Foken, T. (2004) 50 years of the Monin-Obukhov similarity theory. In 16th Symposium on Boundary Layers and Turbulence, Fundamental studies of turbulence: observations, theory, and models session, article 7.1. Portland, ME: Amer. Met. Soc.

García-Díez, M., Fernández, J., Fita, L. and Yagüe, C. (2013) Seasonal dependence of WRF model biases and sensitivity to PBL schemes over Europe. Quarterly Journal of the Royal Meteorological Society, 139, 501-514.

Grachev, A., Fairall, C. W., Persson, P., Andreas, E. L. and Guest, P. (2005) Stable boundary-layer scaling regimes: The Sheba data. Boundary-layer Meteorology, 116, 201-235.

hing, S.-E., Batchvarova, E., Brümmer, B., Jørgensen, H. and Larsen, S. (2007) On the extension of the wind profile over homogeneous terrain beyond the surface boundary layer. Boundary-Layer Meteor., 124, 371-379.

Hartmann, D. L. (ed.) (1994) Global Physical Climatology, vol. 56 of International Geophysics. Academic Press.

Hess, G. D. and Garratt, J. R. (2002) Evaluating models of the neutral, barotropic planetary boundary layer using integral measures. Part I: Overview. Boundary-Layer Meteor., 104, 359-369.

Honst, T. W. (1999) The footprint for estimation of atmosphere-surface exchange fluxes by profile techniques. Boundary-Layer Meteor., 90, 171-188.

Kelly, M. and Gryning, S.-E. (2010) Long-term mean wind profiles based on similarity theory. Boundary-Layer Meteor., 136, 377390.

Kelly, M., Larsen, G., Dimitrov, N. K. and Natarajan, A. (2014a) Probabilistic meteorological characterization for turbine loads. Journal of Physics: Conference Series, 524, 012076. 
Kelly, M. and Troen, I. (2016) Probabilistic stability and "tall" wind profiles: theory and method for use in wind resource assessment. Wind Energy, 19, 227-241.

Kelly, M., Troen, I. and Jørgensen, H. E. (2014b) Weibull-k revisited: "tall" profiles and height variation of wind statistics. Boundary-Layer Meteor., 152, 107-124.

Liu, S. and Liang, X.-Z. (2010) Observed diurnal cycle climatology of planetary boundary layer height. J. Climate, 23, 5790-5809.

Nieliwstadt, F. T. M. (1984) The turbulent structure of the stable, nocturnal boundary layer. Journal of the Atmospheric Sciences, 41, 2202-2216.

Optis, M., Monahan, A. and Bosveld, F. C. (2014) Moving beyond Monin-Obukhov similarity theory in modelling wind-speed profiles in the lower atmospheric boundary layer under stable stratification. Boundary-Layer Meteor., 153, 497-514.

Otte, M. J. and Wyngaard, J. C. (2001) Stably stratified interfacial-layer turbulence from large-eddy simulation. J. Atmos. Sci., 58, 3424-2442.

Pedersen, J. G., Gryning, S.-E. and Kelly, M. (2014) On the structure and adjustment of inversion-capped neutral atmospheric boundary-layer flows: Large-eddy simulation study. Boundary-Layer Meteor., 153, 43-62.

Pedersen, J. G., Kelly, M. and Gryning, S.-E. (2013) The effect of unsteady and baroclinic forcing on predicted wind profiles in large eddy simulations: Two case studies of the daytime atmospheric boundary layer. Meteorologische Zeitschrift, $\mathbf{2 2}$ 661-674.

Sorbjan, Z. (1996) Effects caused by varying the strength of the capping inversion based on a large eddy simulation model of the shear-free convective boundary layer. Journal of the Atmospheric Sciences, 53, 2015-2024.

Sullivan, P. P., Moeng, C.-H., Stevens, B., Lenschow, D. H. and Mayor, S. D. (1998) Structure of the entrainment zone capping the convective atmospheric boundary layer. J. Atmos. Sci., 55, 3042-3064.

Sullivan, P. P. and Patton, E. G. (2011) The effect of mesh resolution on convective boundary layer statistics and structures generated by large-eddy simulation. J. Atmos. Sci., 68, 2395-2415.

Sullivan, P. P., Weil, J., Patton, E. G., Jonker, H. J. and Mironov, D. V. (2016) Turbulent winds and temperature fronts in large-eddy simulations of the stable atmospheric boundary layer. Journal of the Atmospheric Sciences, 73, 1815-1840.

Tennekes, H. and Driedonks, A. (1981) Basic entrainment equations for the atmospheric boundary-layer. Boundary-layer Meteorology, 20, 515-531.

Troen, I. and Petersen, E. L. (1989) European Wind Atlas. Roskilde, Denmark: Risø National Laboratory.

Wyngaard, J. (1973) On surface-layer turbulence. In Workshop on Micrometeorology, 101-149. Duane A. Haugen, Ed.: Amer. Meteor. Soc.

Wyngaard, J. C. (2010) Turbulence in the Atmosphere. Cambridge University Press.

Zilitinkevich, S. and Calanca, P. (2000) An extended similarity theory for the stably stratified atmospheric surface layer. Quarterly Journal of the Royal Meteorological Society, 126, 1913-1923.

Zilitinkevich, S. S. and Esau, I. N. (2002) On integral measures of the neutral barotrophic planetary boundary layer. BoundaryLayer Meteor., 104, 371-379.

- (2005) Resistance and heat-transfer laws for stable and neutral planetary boundary layers: old theory advanced and reevaluated. Quart. J. Roy. Meteor. Soc., 131, 1863-1892.

Zilitinkevich, S. S., Tyuryakov, S. A., Troitskaya, Y. I. and Mareev, E. A. (2012) Theoretical models of the height of the atmospheric boundary layer and turbulent entrainment at its upper boundary. Izv. Atmos. Ocean Phys., 48, 133-142. 